\title{
Recombinant Water Stress Protein 1 (Re-WSP1) Suppresses Colon Cancer Cell Growth Through Mir- 539/B-Catenin Signaling Pathway
}

\section{Songjia Guo}

Shanxi Provincial Peoples Hospital

Shuhua Shan

Shanxi University

Haili Wu

Shanxi University

huiqiang hao ( $\sim$ hhqhyy@163.com )

Shanxi Medical University https://orcid.org/0000-0002-5254-3782

Zhuoyu Li

Shanxi University

\section{Research Article}

Keywords: Re-WSP1, Nostoc commune Vauch, microRNA-539, $\beta$-catenin, Colon cancer

Posted Date: March 13th, 2021

DOl: https://doi.org/10.21203/rs.3.rs-302966/v1

License: (c) (1) This work is licensed under a Creative Commons Attribution 4.0 International License. Read Full License 


\section{Abstract}

Nostoc commune Vauch is a nitrogen-fixing blue-green algae, contains a large number of active molecules with medicinal functions. Our previous study found that a water stress protein (WSP1) from Nostoc commune Vauch and its the recombinant protein (Re-WSP1) exhibited significant anti-colon cancer (CRC) activity both in vitro and in vivo. However, the underlying mechanism remains unknown. In this study, the CCK8 and clonogenic assays showed that Re-WSP1 restrained the colon cancer growth in a dose-dependent manner. Mechanistically, Re-WSP1 inhibited the expression of $\beta$-catenin, which was partly reversed by $\mathrm{LiCl}$ treatment, demonstrating a key role in Re-WSP1-induced inhibition of cell growth. Quantitative PCR analysis showed that the expression of microRNA-539 (miR-539) was significantly upregulated upon Re-WSP1 treatment. Moreover, miR-539 negatively regulateed the expression of $\beta$-catenin through directly binds to the $3^{\prime} \mathrm{UTR}$ of $\beta$-catenin mRNA. Taken together, our data demonstrate that ReWSP1 suppresses the CRC growth via miR-539/ $\beta$-catenin axis, which provides new insights into the molecular mechanisms underlying Re-WSP1 against CRC.

\section{Introduction}

Nostoc commune Vauch, also known as landraces, is a nitrogen-fixing blue-green algae, which is rich in nutrients, including amino acids, proteins, lipids, vitamins, minerals and pigments. Potts et al ${ }^{(1)}$ reported on a set of acidic proteins of the Nostoc commune Vauch, these proteins are synthesized under natural conditions of constant drying and rehydration, because this kind of protein is involved in the water stress response of Nostoc commune Vauch, Potts named it water stress protein (WSP). Water stress protein is the main component of the extracellular matrix ${ }^{[2]}$. Our preliminary study firstly reported that the natural soil water stress protein (WSP1) had significant anti-CRC effect in vitro and in vivo. Then the protein was recombinant expressed by genetic engineering technology to obtain recombinant water stress protein (ReWSP1). The in vitro experimental data showed that the Re-WSP1 has significant anti-colon cancer effect. However, its mechanism of anti-colon cancer effect still needs further research.

Colorectal cancer (CRC) is one of the most common malignancies in the world, with the incidence of males and females occupying the third and the second place respectively ${ }^{[3]}$. Activation of $\mathrm{Wnt} / \beta$-catenin signaling pathway plays an important role in the development and progression of CRC. Studies have shown that more than $90 \%$ of human CRC have aberrant activation of Wnt/ $\beta$-catenin signaling pathway, accompanying accumulation of intracellular $\beta$-catenin ${ }^{[4]}$. With the rapid development of molecular oncology and molecular pharmacology, a new development strategy for finding active ingredients in today's anti-cancer drugs has emerged from natural products.

MicroRNAs (miRNAs) are endogenous non-coding RNAs that regulate gene expression via binding to the 3'-untranslated region (UTR) of a specific messenger RNA (mRNA), resulting in either mRNA degradation or translational inhibition; miRNAs are small, being only $18-25$ nucleotides in length ${ }^{[5,6]}$. Emerging evidence suggests that miRNAs are related to development and progression of CRC as either tumor suppressors or oncogenes ${ }^{[7,8]}$. 
In present study, our result demonstrates that Re-WSP1 suppresses the CRC growth via miR-539/ $\beta$ catenin axis, which provides new insights into the molecular mechanisms underlying Re-WSP1.

\section{Materials And Methods \\ 2.1. Cell lines}

The human colon cancer cell HT29 and DLD1 were obtained from the American Type Culture Collection (ATCC, Rockville, MD, USA) and cultured in RPIM-1640 medium (Gibco, Grand Island, NY, USA) supplemented with $10 \%$ heat-inactivated fetal bovine serum (FBS, Evergreen), $100 \mathrm{U} / \mathrm{mL}$ penicillin, and $100 \mathrm{mg} / \mathrm{mL}$ streptomycin in a humidified chamber supplemented with $5 \% \mathrm{CO}_{2}$ at $37^{\circ} \mathrm{C}$.

\subsection{Expression and Purification of Recombinant Protein Re- WSP1}

Primers were designed according to the coding sequence of WSP1 gene, six histidine His tags were added at the $\mathrm{N}$ end of WSP1. At the two ends of the primers, enzyme digestion sites BamH I and Xho I were introduced, and primers were synthesized by Takara Company. CDNA was used as the template for the amplification by PCR technology, and then the PCR products were double-zapped with restriction enzyme Xhol and BmaH I. After gel recovery, the target fragment was connected with pet-28a. The constructed vector was transformed into E. coli DH5a. Positive recombinants were selected with LB plates containing kanamycin. Single colonies were verified by PCR, and the selected positive recombinants were sequenced by Shanghai Sangon. The recombinant plasmid was named Re-WSP1-pet28a and transformed into the expression system of E.coli BL21 (DE3) cells. The transformed cells were grown in LB agar containing kanamycin. Bacteria were cultured in LB medium containing kanamycin. When the bacteria reached logarithmic growth stage $(A 600=0.5)$, the recombinant vector plasmid was induced by IPTG, and the control group without IPTG. Then the bacterial cells were collected by centrifuge and subjected to ultrasonication. The supernatant was filtered with $0.45 \mu \mathrm{m}$ filter for later use. Separation and purification were preformed according to the instructions for affinity chromatography. After obtaining the target protein, detected by SDS-PAGE electrophoresis.

\subsection{Cell proliferation and colony formation analyses}

Cell proliferation was determined using a Cell Counting Kit-8 assay (Dojindo Laboratories, Kumamoto, Japan). Briefly, cells were seeded into a 96-well plate at a density of $5 \times 10^{3}$ cells/well, and then exposed to different concentrations $(0,0.05,0.1,0.15$, and $0.2 \mu \mathrm{g} / \mu \mathrm{L})$ of Re-WSP1 for $24 \mathrm{~h}$. At the indicated time, $20 \mu \mathrm{L} \mathrm{CCK8} \mathrm{reagent} \mathrm{was} \mathrm{added} \mathrm{into} \mathrm{each} \mathrm{well} \mathrm{and} \mathrm{incubated} \mathrm{for} 4 \mathrm{~h}$ at $37^{\circ} \mathrm{C}$. The absorbance at a wavelength of $450 \mathrm{~nm}$ was detected using a Benchmark PlusTM microplate spectrometer (Bio-Rad, USA).

For the colony formation assay, cells were seeded into a 6-well plate at density of 1000 cells/well and cultured for two weeks. Cell colonies were then fixed with $10 \%$ formaldehyde (Sigma, USA), stained with $0.1 \%$ crystal violet (Sigma), and counted using a microscope (Olympus, Tokyo, Japan). 


\subsection{Western blot}

CRC cells were harvested and lysed with RIPA buffer (Beyotime, Jiangsu, China) followed by quantification with BCA Protein Assay Kit (Pierce, Bonn, Germany). The protein expression was determined by western blot as described previously ${ }^{[9]}$. The following primary antibodies were used in this study: $\beta$-catenin (1:1000, Santa Cruz, CA, USA), c-Myc (1:1000, Santa Cruz, CA, USA), cyclin D (1:1000, Santa Cruz, CA, USA) and GAPDH (1:3000, Santa Cruz). GAPDH was used as a control.

\subsection{RNA extraction and quantitative PCR (qPCR)}

Total RNA from HT29 and DLD1 were isolated using the mirVana miRNA Isolation Kit (Ambion, Carlsbad, CA, USA), and qPCR was performed using the microRNA Assay Kit (Ambion) and an Applied Biosystems 7900 HT system (Applied Biosystems, CA, USA), with the U6 primer as an internal control. The expression level was calculated by the $2^{-\triangle \Delta C t}$ method.

\subsection{The dual luciferase reporter assay}

The $\beta$-catenin 3'UTR was amplified by PCR and subcloned into the psiCHECK2.0 vector (Ambion, Austin, TX, USA) and the resulting plasmid named $\beta$-catenin-3'UTR. For the luciferase reporter assay, HT29 cell were co-transfected with miR-539 mimic (GenePharma Co., Shanghai, China), corresponding negative control mimic (miR-NC, GenePharma), miR-539 inhibitor (GenePharma) and negative control inhibitor (NC inhibitor, GenePharma) using Lipofectamine 2000. The relative luciferase activity was determined using a Dual-Luciferase ${ }^{\circledR}$ Reporter Assay Kit (Promega, Madison, WI, USA) according to the manufacturer's protocol after $48 \mathrm{~h}$ transfection. Renilla luciferase activity was normalized to firefly luciferase activity.

\subsection{Statistical analysis}

All statistical analyses were carried out using the SPSS statistical software for Windows Version 20 (SPSS, Chicago, IL, USA). Values are expressed as the mean \pm standard deviation (SD) from at least three independent experiments. Comparisons between the groups were analyzed by Student's T-test or a oneway ANOVA with Dunn's least significant difference test. In all cases, $\mathrm{P}<0.05$ was considered to indicate statistical significance.

\section{Results}

\subsection{Re-WSP1 inhibits proliferation and colony formation in CRC cells.}

To assess the biological effects of Re-WSP1 on CRC cells, CCK8 and colony formation were performed. The results demonstrated that the cell viability gradually decreased along with the increase of Re-WSP1 concentration, and the number of cell clones in Re-WSP1-treatment group was largely lower than that in control group (Fig. 1A and 1B). 


\section{2 $\beta$-catenin mediates the inhibitory effect of Re-WSP1 on CRC cells.}

It's reported that aberrant activation of $\beta$-catenin signaling pathway plays an important role in the development and progression of colon cancer. Then we detected the expression levels of $\beta$-catenin and its downstream factors c-Myc and cyclin D with different concentrations of Re-WSP1 treatment. The results showed that Re-WSP1 treatment decreased the expression levels of $\beta$-catenin, c-Myc and cyclin D in HT-29 and DLD1 cells (Fig. 2A and 2B). To further validate the involvement of $\beta$-catenin in Re-WSP1induced CRC cells growth inhibition, cells were treated with the activator of Wnt signaling pathway ( $\mathrm{LiCl})$. It was found that $\mathrm{LiCl}$ treatment markedly reversed the expression levels of $\beta$-catenin, $\mathrm{C}-\mathrm{Myc}$ and cyclin $\mathrm{D}$, indicating that $\beta$-catenin mediates the inhibition effect of Re-WSP1 on CRC cells (Fig. 2C and 2D).

\section{3 miR-539 directly inhibits $\beta$-catenin expression}

It is known that the expression of $\beta$-catenin is regulated by miRNAs at the post-transcription level. By screening the databases miRanda and TargetScan, it was found that miR-543, miR-212, miR-539, miR132 , miR-129 and miR-221 conserved to target $\beta$-catenin, so we detected the expression levels of these miRNAs using qPCR after Re-WSP1 treatment. The results showed that among the above miRNAs, miR539 was significantly increased in a dose dependent manner, and while other miRNAs had no significant change both in HT-29 and DLD1 cells (Fig. 3A and 3B).

To determine the relationship between miR-539 and $\beta$-catenin, after cells were transfected with miR-539 mimic and inhibitor, the expression levels of miR-539 and $\beta$-catenin were detected. The results showed that miR-539 mimic treatment increased miR-539 expression and decreased $\beta$-catenin expression, and while miR-539 inhibitor treatment exhibited an opposite trend (Fig. 3C and 3D), indicating that miR-539 negatively regulates the expression of $\beta$-catenin. Based on the above evidence, we focused on whether miR-539 repressed the expression of $\beta$-catenin by directly binding to the 3'UTR of $\beta$-catenin mRNA. A putative miR-539 binding sites in the $3^{\prime} U T R$ of $\beta$-catenin were predicted through bioinformatics analysis (Fig. 3E). Further using the dual luciferase reporter system to determine whether miR-539 directly regulates $\beta$-catenin, thus constructing a dual luciferase reporter vector of $\beta$-catenin $3^{\prime} U T R$, and then cotransfecting cells with miR-539 mimic and inhibitor, respectively. It was shown that transfected miR-539 mimic showed decreased luciferase activity, and transfected miR-539 inhibitor showed increased luciferase activity, indicating that miR-539 can directly target $\beta$-catenin $3^{\prime} U T R$, thereby inhibiting the expression of $\beta$-catenin (Fig. 3F).

\subsection{The role of miR-539 in growth inhibition induced by Re- WSP1<}

To verify the role of miR-539 in the growth inhibition induced by Re-WSP1, cells were treated with ReWSP1 and miR-539 inhibitor cotreatment, and found that the cell survival rate remarkably increased compared with the single plus Re-WSP1 group. This indicates that the cell growth inhibition induced by 
Re-WSP1 can be partly neutralized transfected by miR-539 inhibition (Fig. 4A). Further, after cells were transfected by miR-539 inhibition, western blot results showed that the decreased expression of $\beta$-catenin induced by Re-WSP1 was also significantly reversed indicating that miR-539 mediates the inhibition effect of $\beta$-catenin induced by Re-WSP1 (Fig. 4B).

\section{Discussion}

The land vegetable is a combination of fungi and algae, generally growing in dark and humid places, dark black, a bit like soft Black fungus. Scientists from the Weizmann Institute of Israel have found that a component of the genus Dioscorea could inhibit the activity of acetylcholinesterase in the human brain, thereby producing a therapeutic effect on Alzheimer's disease [10]. Our results confirmed that Re-WSP1 could inhibit the proliferation and colonization of CRC cells (Fig. 1) and the expression of miR-539 in CRC cells notably increased with the increased Re-WSP1 concentration treatment (Fig. 4). These results indicate that the anti-CRC effects Re-WSP1 is associated with the expression of miR-539.

We know that $\beta$-catenin is a critical component of the well-studied Wnt/ $\beta$-catenin signaling pathway. Studies have shown that the abnormal expression of $\beta$-catenin is positively correlated with the conversion of colon adenoma to CRC [11-13].

miRNAs participate in multiple biological processes, including metabolic homeostasis, cell proliferation, invasion, the cell cycle and apoptosis ${ }^{[14,15]}$. In papillary thyroid carcinoma, the up-regulation of miR-155 promotes tumor cell invasion by activating $\beta$-catenin ${ }^{[16]}$. In neuroblastoma, miR-155 is able to promote disease progression by activating $\beta$-catenin ${ }^{[17]}$. In addition, hepatitis virus can also regulate the $\mathrm{Wnt} / \beta$ catenin signaling pathway by up-regulating the expression of miR-155, thereby initiating liver cancer ${ }^{[18]}$. However, in CRC, whether miR-539 is involved in the pathogenesis and its role in the proliferation of CRC cells has not been reported yet. In present study, we found that miR-539 negatively regulated the expression of $\beta$-catenin by directly binding to $\beta$-catenin $3^{\prime} U T R$, implying a tumor suppressor role in CRC.

In summary, we preliminarily established that miR-539 played an important role in the anti-CRC effect induced by Re-WSP1 in CRC cells. Re-WSP1 negatively regulated the expression of $\beta$-catenin by upregulating the expression level of miR-539, and then participated in the regulation of $\beta$-catenin signaling pathway, resulting in the growth inhibition of of CRC cells. It disclosed a novel mode of ReWSP1 toxicity to CRC cells mediated by miR-539and shed light on the function of miR-539 in the regulation of CRC cell proliferation. This might open up some new possibilities for Re-WSP1 as future therapeutic intervention.

\section{Declarations}

\section{Funding}


This study was supported by National Natural Science Foundation of China (No. 81603020, 31770382).

\section{Conflicts of interest}

The authors declare no conflict of interest.

\section{Availability of data and material}

The datasets used or analysed during the current study are available from the corresponding author on reasonable request.

\section{Code availability}

Not applicable.

\section{Authors' contributions}

All the authors have contributed to the collection of references, the contents of the articles and charts.

\section{Ethics approval and Consent to participate}

Not applicable.

\section{Consent for publication}

Not applicable.

\section{References}

1. SCHERER S, POTTS M (1989) Novel water stress protein from a desiccation-tolerant cyanobacterium. Purification and partial characterization [J]. J Biol Chem 264(21):12546-12553

2. SAKAMOTO T, KUMIHASHI K, KUNITA S et al (2011) The extracellular-matrix-retaining cyanobacterium Nostoc verrucosum accumulates trehalose, but is sensitive to desiccation [J]. FEMS Microbiol Ecol 77(2):385-394

3. Boyle P, Leon ME (2002) Epidemiology of colorectal cancer [J]. Br Med Bull 64(474):1-25

4. Yoshida N, Kinugasa T, Ohshima K et al (2015) Analysis of Wnt and $\beta$-catenin Expression in Advanced Colorectal Cancer [J]. Anticancer Res 35(8):4403-4410

5. Valinezhad Orang A, Safaralizadeh R, Kazemzadeh-Bavili M, Mechanisms of miRNA-Mediated gene regulation from common downregulation to mRNA-Specific upregulation[J]. Int. J. Genomics 2014, 970607

6. Hwang HW, Mendell JT (2007) MicroRNAs in cell proliferation, cell death, and tumorigenesis[J]. $\mathrm{Br} \mathrm{J}$ Cancer 96:R40-R44 
7. Dong Y, Yu J, Ng SS, MicroRNA dysregulation as a prognostic biomarker in colorectal cancer[J]. Cancer Manage. Res.2014,6: 405-422

8. Zhou JJ, Zheng S, Sun LF, Zheng L (2014) MicroRNA regulation network in colorectal cancer metastasis[J]. World J Biol Chem 5:301-307

9. Wang P, Meng X, Huang Y, Lv Z, Liu J, Wang G, Meng W, Xue S, Zhang Q, Zhang P, Chen G (2017) MicroRNA-497 inhibits thyroid cancer tumor growth and invasion by suppressing BDNF[J]. Oncotarget 8:2825-2834

10. Olarewaju OA, Alashi, Adeola M et al (2018) Influence of nitrogen fertilizer micro-dosing on phenolic content, antioxidant, and anticholinesterase properties of aqueous extracts of three tropical leafy vegetables[J]. J Food Biochem 42(4):1. Doi:10.1111/jfbc.12566

11. Chen J-S;Huang Jiong-Qiang;Luo Bing,et al. PIK3CD induces cell growth and invasion by activating

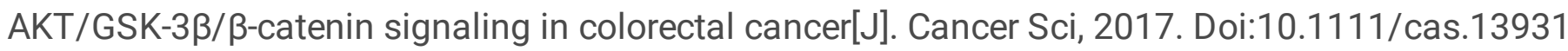

12. Junhui Yu;DongLiu;Xuejun, Sun,et al. CDX2 inhibits the proliferation and tumor formation of colon cancer cells by suppressing Wnt/ $\beta$-catenin signaling via transactivation of GSK-3 $\beta$ and Axin2 expression[J]. Cell Death Dis. 2019, (10), doi:10.1038/s41419-018-1263-9

13. Wu Zizhen,Tang Fuqiu,Yang Bo. Ectopic expression of $\beta$-catenin in colon cancer and its effect on the proliferation of colon cancer cells[J].South China Journal of National Defence Medicine,2018,(11). 745-748

14. Bushati N, Cohen SM (2007) microRNA functions[J]. Annu Rev Cell Dev Biol 23:175-205

15. Volinia S, Calin GA, Liu CG et al. A microRNA expression signature of human solid tumors defines cancer gene targets[J]. Proc. Natl. Acad. Sci. U.S. A. 2006,103:2257-2261

16. Zhang Xing L, Miao Z, Kai et al (2013) Upregulated miR-155 in papillary thyroid carcinoma promotes tumor growth by targeting APC and activating Wnt/beta-catenin signaling [J]. J Clin Endocrinol Metab 98(8):E1305-E1313

17. Zhi Y, Shaofei C, Jian W et al (2015) miR-155 contributes to the progression of glioma by enhancing Wnt/beta-catenin pathway[J]. Tumour Biol 36(7):5323-5331

18. Zhang Y, Wuqing W, Na C et al (2012) Hepatitis C virus-induced up-regulation of microRNA-155 promotes hepatocarcinogenesis by activating Wnt signaling [J]. Hepatology 56(5):1631-1640

\section{Figures}


A

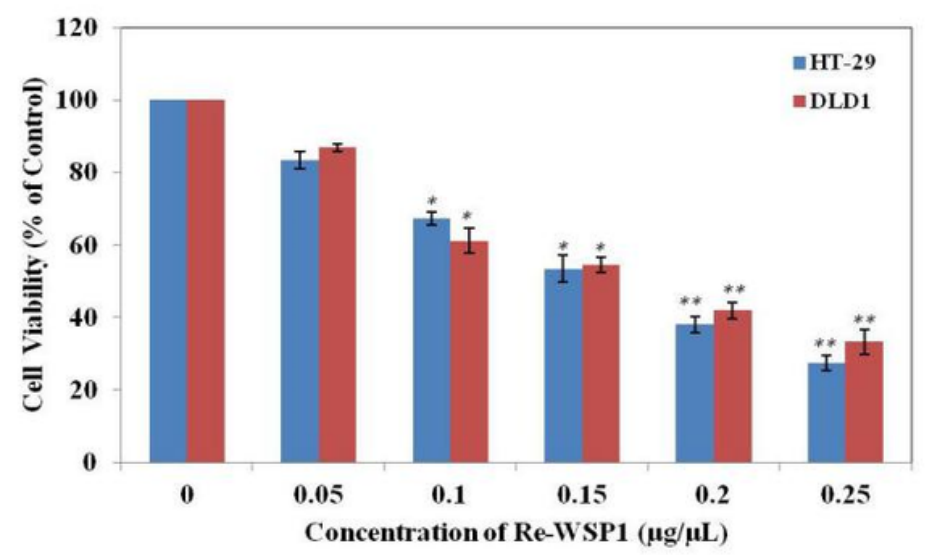

B

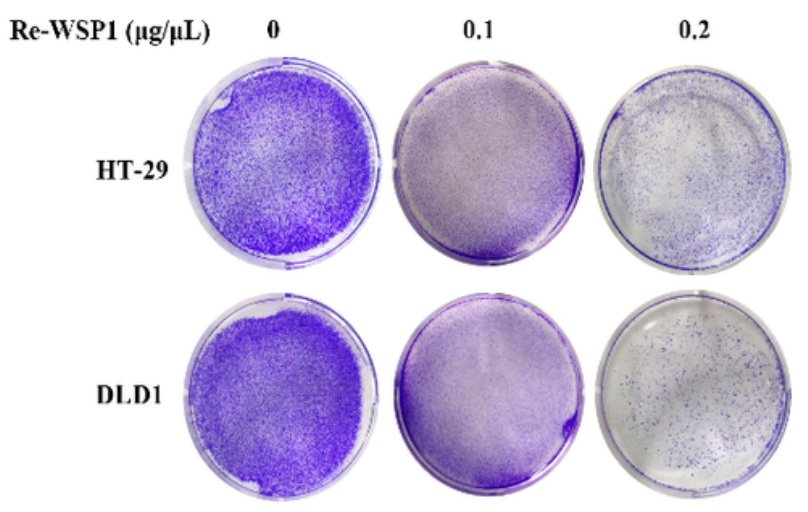

\section{Figure 1}

To assess the biological effects of Re-WSP1 on CRC cells, CCK8 and colony formation were performed. The results demonstrated that the cell viability gradually decreased along with the increase of Re-WSP1 concentration, and the number of cell clones in Re-WSP1-treatment group was largely lower than that in control group 
A.

\section{$\operatorname{Re}-W S P 1(\mu \mathrm{g} / \mu \mathrm{L})$}
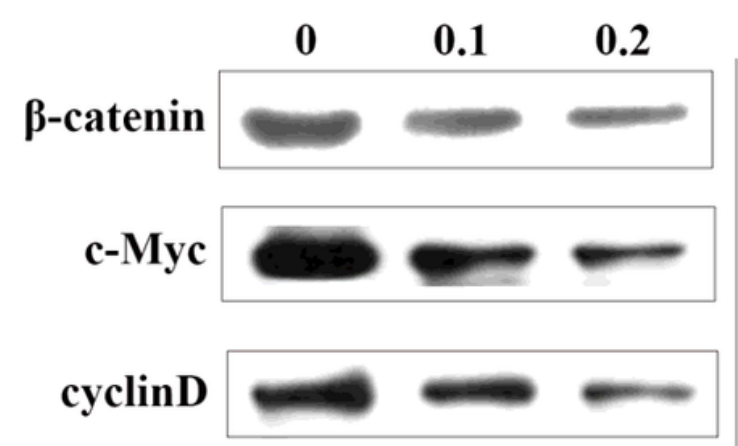

Эิ

\section{GAPDH}

C

HT-29

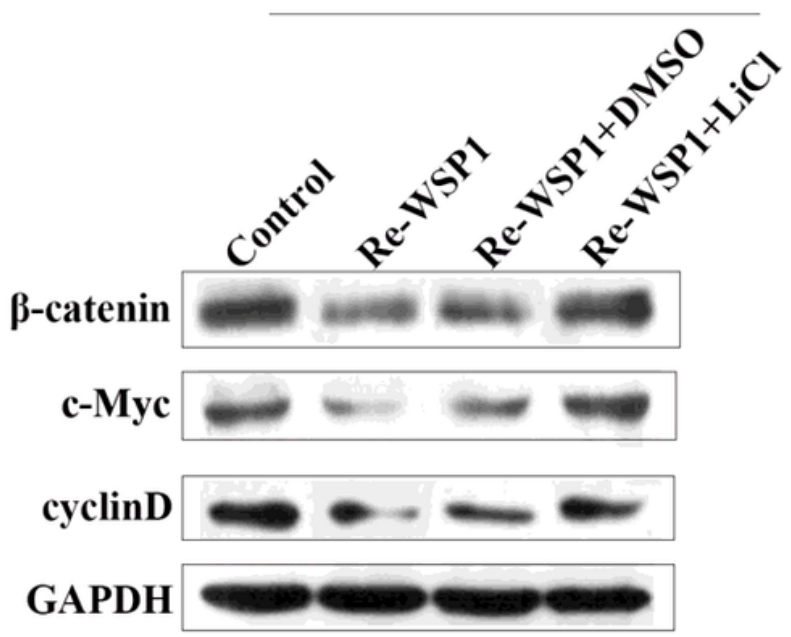

B.

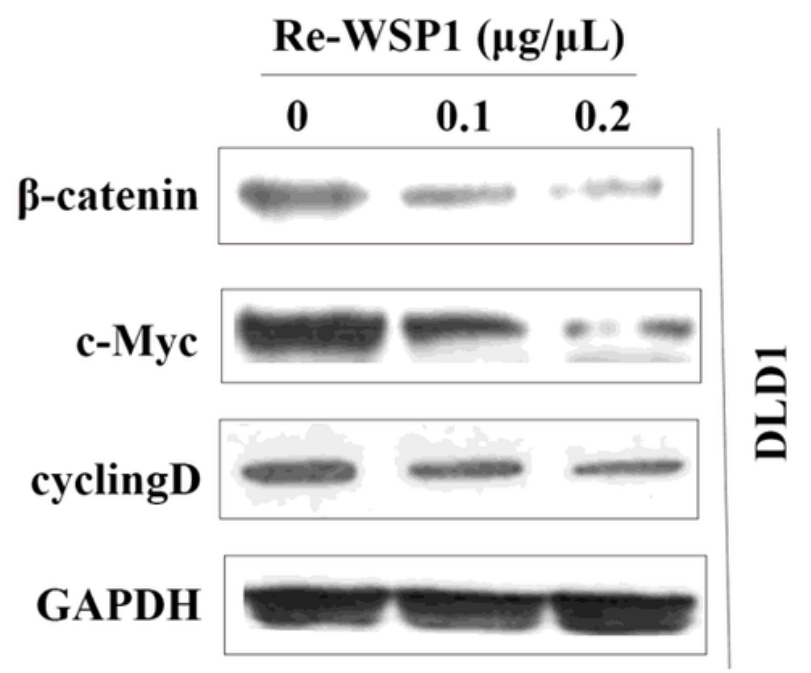

D

DLD1

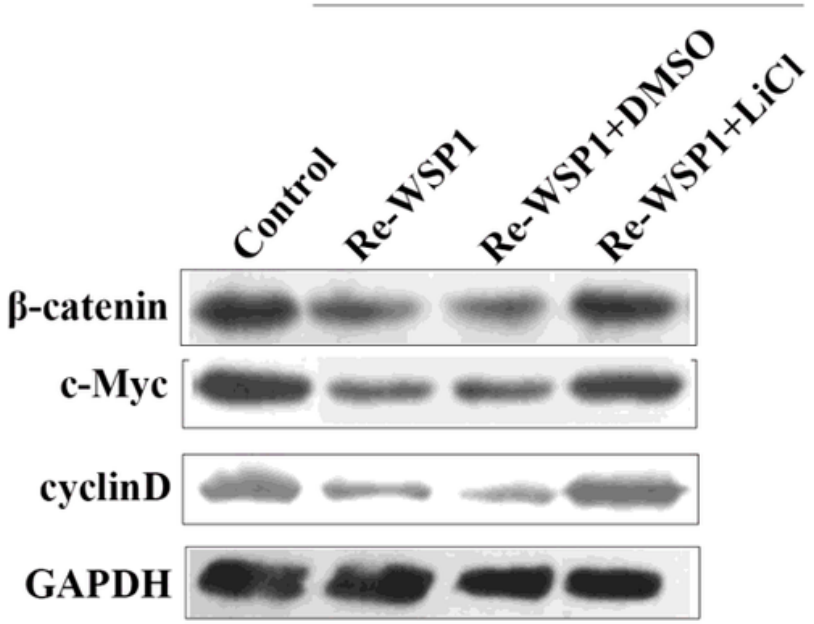

Figure 2

It's reported that aberrant activation of $\beta$-catenin signaling pathway plays an important role in the development and progression of colon cancer. Then we detected the expression levels of $\beta$-catenin and its downstream factors c-Myc and cyclin D with different concentrations of Re-WSP1 treatment. The results showed that Re-WSP1 treatment decreased the expression levels of $\beta$-catenin, c-Myc and cyclin D in HT-29 and DLD1 cells 
A

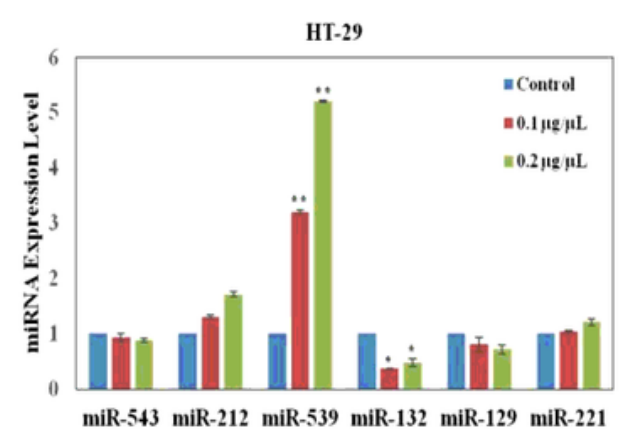

C

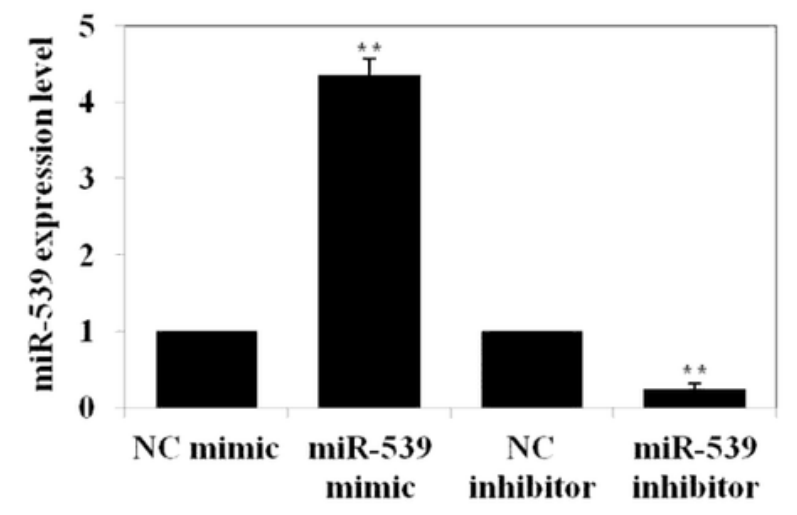

$\mathbf{E}$

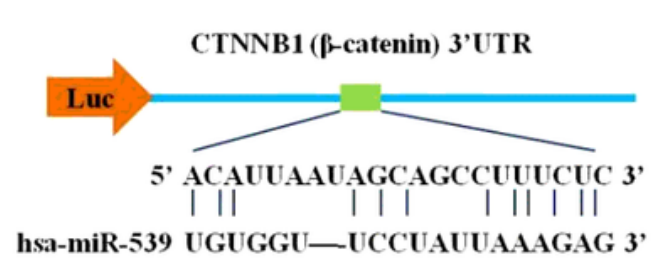

B

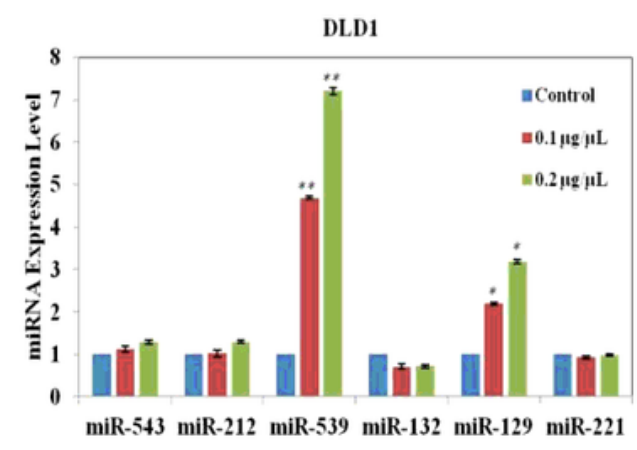

D

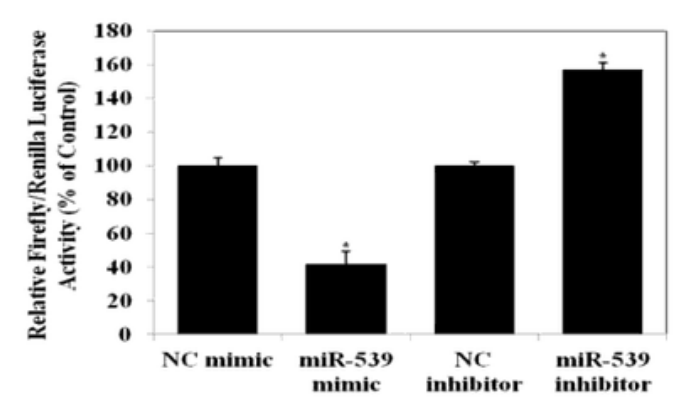

F

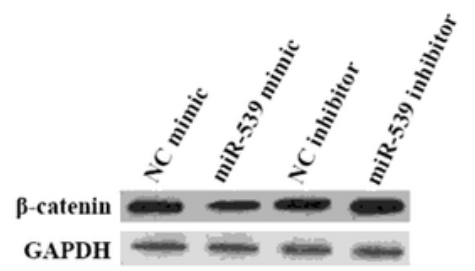

\section{Figure 3}

It is known that the expression of $\beta$-catenin is regulated by miRNAs at the post-transcription level. By screening the databases miRanda and TargetScan, it was found that miR-543, miR-212, miR-539, miR132 , miR-129 and miR-221 conserved to target $\beta$-catenin, so we detected the expression levels of these miRNAs using qPCR after Re-WSP1 treatment. The results showed that among the above miRNAs, miR- 
539 was significantly increased in a dose dependent manner, and while other miRNAs had no significant change both in HT-29 and DLD1 cells

A

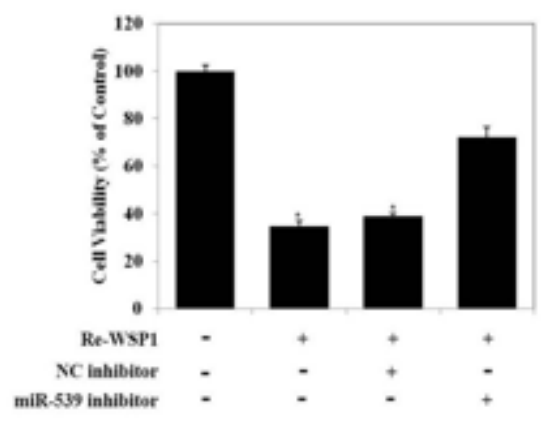

B

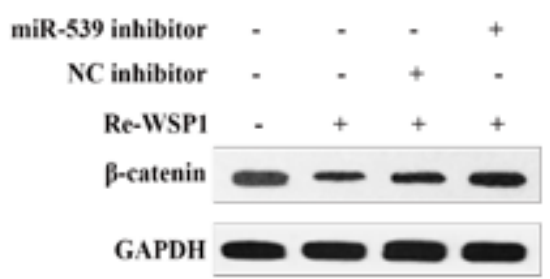

B.

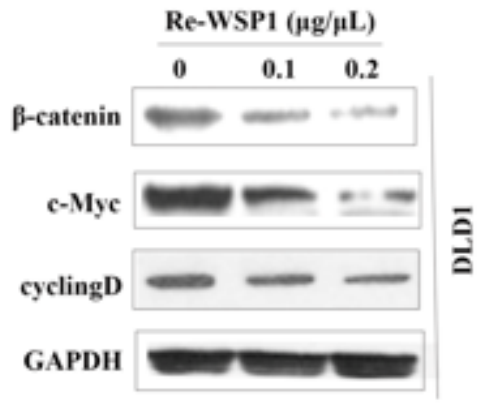

D

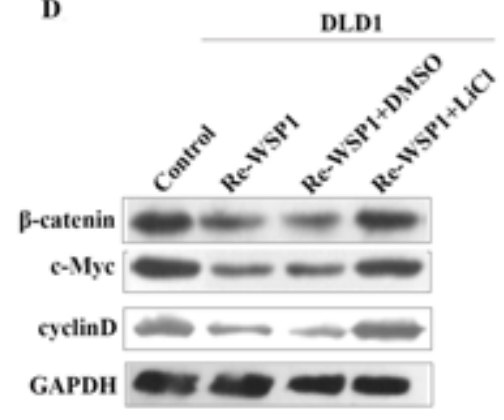

\section{Figure 4}

Further, after cells were transfected by miR-539 inhibition, western blot results showed that the decreased expression of $\beta$-catenin induced by Re-WSP1 was also significantly reversed indicating that miR-539 mediates the inhibition effect of $\beta$-catenin induced by Re-WSP1 


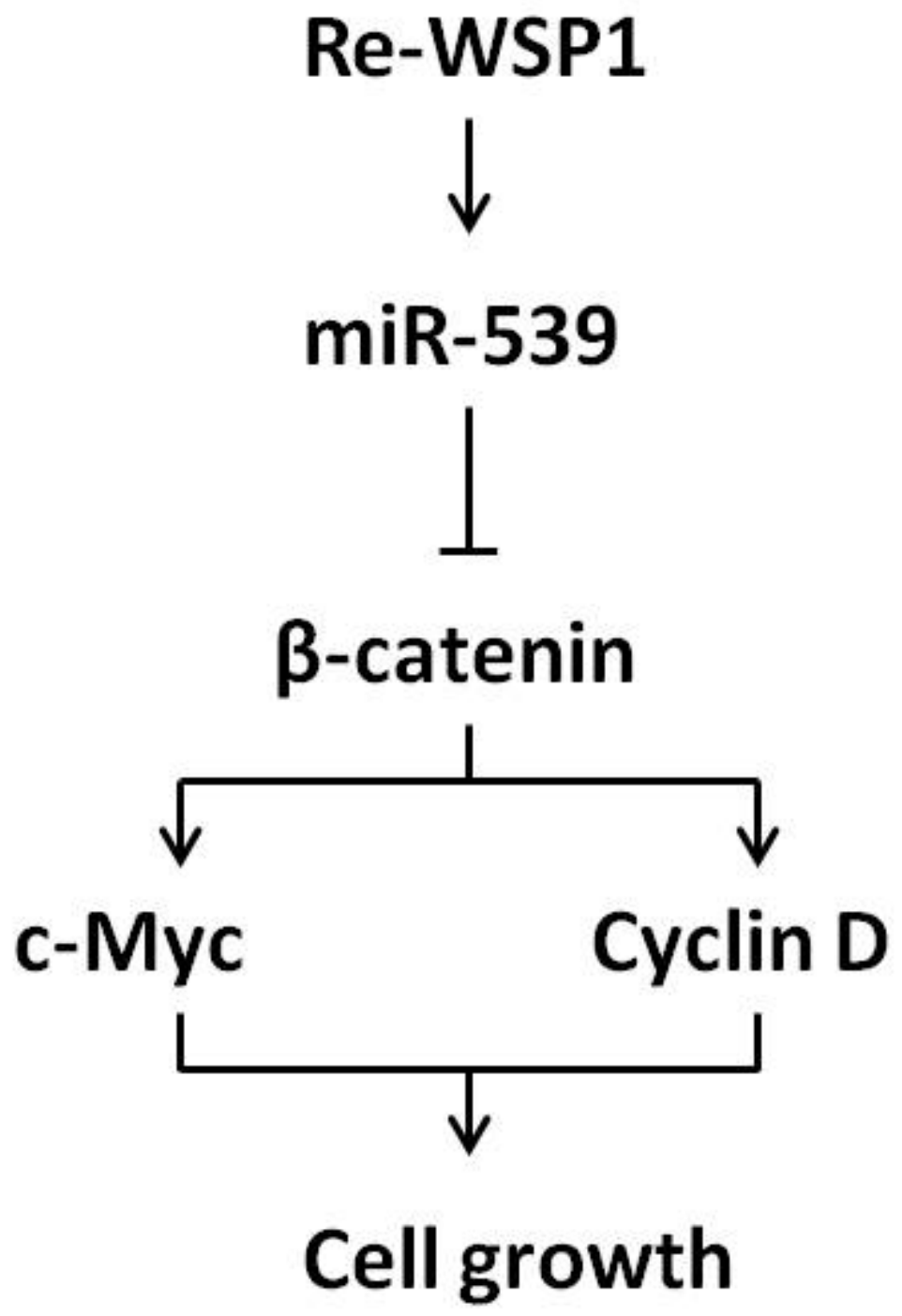

Figure 5

Flowchart of the study 